\title{
Risk factors of postoperative pulmonary complications following elective craniotomy for patients with tumors of the brainstem or adjacent to the brainstem
}

\author{
HUI CHU and BIN-WEN DANG \\ Department of Respiratory Medicine, Beijing Tiantan Hospital, Capital Medical University, Beijing 100050, P.R. China
}

Received December 12, 2013; Accepted June 1, 2014

DOI: $10.3892 / \mathrm{ol} .2014 .2374$

\begin{abstract}
The aim of the present study was to analyze the risk factors of postoperative pulmonary complications (PPCs) of elective craniotomy for patients presenting with brainstem tumors or tumors adjacent to the brainstem. A total of 162 consecutive patients with a brainstem tumor or adjacent brainstem tumor undergoing elective craniotomy were included and monitored. Potential risk factors were identified by data collection and monitoring of the PPCs, as well as the performance of single factor analysis (using the $\chi^{2}$ test). In addition, the independent risk factors of PPCs were screened by logistic analysis. A total of 39 cases of PPC were included in the current study, with an incidence rate of $23.9 \%$. The analysis indicated that smoking history, previous pulmonary diseases, an American Society of Anesthesiologists classification >II and partial tumor resection were risk factors of PPC following an elective craniotomy. Smoking history and partial tumor resection were identified to be independent risk factors of PPCs.
\end{abstract}

\section{Introduction}

Postoperative pulmonary complications (PPCs) refers to the incidence of postoperative clinical manifestations of pulmonary abnormalities. These complications have a negative effect on disease progression, resulting in patients undergoing prolonged periods of hospitalization in addition to increasing the rate of postoperative mortality (1). PPCs predominantly include pneumonia, bronchitis, atelectasis, deterioration of lung disease and respiratory failure, which together account for $11.2-24.6 \%$ of all pulmonary-associated complications $(2,3)$. Brainstem tumors refer to tumors that occur in the brainstem, including the midbrain, pons and medulla oblongata, and

Correspondence to: Mr. Bin-Wen Dang, Department of Respiratory Medicine, Beijing Tiantan Hospital, Capital Medical University, 6 Tiantan Xili, Dongcheng, Beijing 100050, P.R. China

E-mail: binwudang@126.com

Key words: brain tumor, craniotomy, postoperative pulmonary complications, risk factors adjacent brainstem tumors refer to the tumors that occur in the area surrounding the brainstem, invading and oppressing the brainstem, and affecting brainstem function. A craniotomy for this type of tumor has different features when compared with other craniocerebral surgical procedures, which may be due to the specific location of the tumor, which results in a complex and risky procedure (4). Clinical findings of PPCs are well known, however, the incidence rate and associated risk factors are not often reported. Therefore, it is considered to be necessary to independently investigate this type of craniocerebral surgical procedure. The present prospective study was performed at the Beijing Tiantan Hospital (Beijing, China). This hospital includes a Neurosurgery Institute, in which the brainstem ward has 42 beds, predominantly for patients admitted for surgical intervention of brainstem or adjacent brainstem tumors, therefore, providing a large number of patients for inclusion in the present study. A prospective study was therefore conducted, which aimed to identify risk factors of PPCs that are commonly observed in patients following surgical intervention of brainstem tumors, and to facilitate with reducing the incidence of PPCs.

\section{Subjects and methods}

Subjects. A total of 162 consecutive patients with a brainstem tumor or adjacent brainstem tumor who underwent an elective craniotomy between August 2012 and February 2013, were included in the present study. This study was conducted in accordance with the Declaration of Helsinki and with approval from the Ethics Committee of the Beijing Tiantan Hospital, Capital Medical University (Beijing, China). Written informed consent was obtained from all participants.

Methods. A senior physician of the Department of Respiratory Medicine performed all patient examinations and data collection. The director of the department was consulted to resolve any queries. Routine preoperative patient evaluations were conducted to obtain the following data: Patient age, gender, smoking history, body mass index, routine blood analysis, blood glucose and serum albumin levels, liver and kidney function, tumor location, imaging data and the American Society of Anesthesiologists (ASA) physical status classification grade. Postoperatively, the anesthetic methods and medicines, surgery duration, postoperative pathological diagnosis, tumor size 
Table I. Type and incidence of PPCs in the cohort of 162 patients.

\begin{tabular}{lrrr}
\hline & & & Incidence, $\%$ \\
\cline { 2 - 4 } PPC & Cases, $\mathrm{n}$ & $\begin{array}{c}\text { Rate of total } \\
\text { PPCs }\end{array}$ & $\begin{array}{c}\text { Rate of overall } \\
\text { number of patients }\end{array}$ \\
\hline Pneumonia & 20 & 51.3 & 12.3 \\
Bronchitis & 14 & 35.9 & 8.6 \\
Acute respiratory failure & 2 & 5.1 & 1.2 \\
Primary pulmonary disease exacerbation & 2 & 5.1 & 1.2 \\
Atelectasis & 1 & 2.6 & 0.6 \\
Total & 39 & 100 & 23.9 \\
\hline
\end{tabular}

PPC, postoperative pulmonary complication.

and whether a full, near-total or partial tumor resection was conducted was recorded. Postoperative visits for seven consecutive days were performed to monitor the respiratory symptoms and body temperature; in addition, chest X-rays and blood analyses were performed when necessary. These data assisted the neurosurgeon with the diagnosis and treatment of the PPCs.

Diagnostic criteria of the PPCs. PPCs were grouped into five categories as follows: (i) Pneumonia; classified as a recent pulmonary infiltration by chest radiography associated with at least two symptoms including, purulent tracheobronchial secretion, body temperature $>38.3^{\circ} \mathrm{C}$ and $>25 \%$ leukocytes in circulation above the base count $(5,6)$. (ii) Tracheobronchitis; classified as an increase in volume, or a change in the color or purulent aspect of the tracheobronchial secretion with a normal chest radiograph $(5,6)$. (iii) Atelectasis; classified by evidence from a chest radiography of a pulmonary atelectasis with associated acute respiratory symptoms (6,7). (iv) Acute exacerbation of chronic obstructive pulmonary disease (COPD); an event in the natural course of the disease characterized by a worsening of the patient's baseline dyspnea, a cough and/or sputum, which is outside of the normal variability that is sufficient to warrant a change in treatment management. In cases where a chest radiograph indicated shadowing, consistent with infection, the patient was considered to have pneumonia (8). (v) Respiratory failure; characterized by a shortness of breath, dyspnea, respiratory frequency of $>25$ beats/min, with blood gas analysis (with no oxygen) revealing a partial pressure of $\mathrm{O}_{2}\left(\mathrm{PaO}_{2}\right)<60 \mathrm{mmHg}$ and/or partial pressure of $\mathrm{CO}_{2}>50 \mathrm{mmHg}$, and an $\mathrm{O}_{2}$ inhalation oxygenation index $\left(\mathrm{PaO}_{2} /\right.$ fraction of inspired $\left.\mathrm{O}_{2}\right) \leq 300 \mathrm{mmHg}$.

Statistical analysis. The possible risk factors were statsitically analyzed using SPSS (SPSS, Inc., Chicago, IL, USA). The $\chi^{2}$ test was used for univariate analyses, and statistically significant factors underwent a logistic regression analysis to screen the independent risk factors of PPCs. $\mathrm{P}<0.05$ was considered to indicate a statistically significant difference.

\section{Results}

Subject data. The present study included a total of 162 patients (males, $n=70$; females, $n=92$ ), aged $16-78$ years, with a mean age of $44.33 \pm 1.02$ years. Patients aged $\geq 60$ and $\geq 65$ years accounted for $9.26 \%$ and $1.85 \%$, respectively of the total population. The patients exhibited normal preoperative hepatic function, routine blood test results, renal function, and serum albumin and blood sugar levels. No serious cardiopulmonary insufficiencies (ASA class >III patients, $\mathrm{n}=0$; ASA class III patients, $\mathrm{n}=6$; COPD patients, $\mathrm{n}=4$; heart disease patients, $n=2$; senile valve disease patients, $n=1$; coronary heart disease patient, $n=1$ ) and no patients with a history of malignant tumors were identified. A total of 25 cases had a history of smoking and continued to smoke eight weeks prior to surgery.

Distribution of tumor locations. There were 68 cases of brainstem tumors and 94 cases of adjacent brainstem tumors. There were 121 cases of benign tumors, 41 cases of malignant tumors, 59 cases of meningioma, 32 cases of neurilemmoma, 17 cases of glioma, 10 cases of chordoma, 10 cases of cavernous hemangioma, seven cases of hemangioblastoma, seven cases of ependymoma, six cases of astrocytoma, six cases of epidermoid cyst, four cases of glomus jugular tumor and four cases of other tumors.

Anesthesia and surgical procedure. All patients underwent a craniotomy under intravenous anesthesia and tracheal intubation with mechanical ventilation, without the use of long-acting neuromuscular blocking agents, such as pancuronium bromide. All surgical procedures were performed by a team of three surgeons, following which the patients were admitted to the Department of Neurosurgery ward. The average surgery duration was $6.46 \pm 0.16 \mathrm{~h}$ (range, 3.00-12.25 h). A total resection was performed in 134 cases and a near-total or partial resection was performed in 28 cases.

Pulmonary complications. There were 39 cases of PPCs, with an incidence of $23.9 \%$ (Table I).

Single factor and logistic regression analysis. Single factor analysis demonstrated an association between PPCs and smoking history, underlying lung disease, ASA class $>$ II and partial tumor resection. A logistic regression analysis on these risk factors indicated that smoking history and partial tumor 
Table II. Single factor analysis of risk factors of PPCs.

\begin{tabular}{|c|c|c|c|c|}
\hline & \multicolumn{2}{|c|}{$\mathrm{PPC}$} & \multirow[b]{2}{*}{$\chi^{2}$ value } & \multirow[b]{2}{*}{ P-value } \\
\hline & Affected, n & Non-affected, n & & \\
\hline \multicolumn{5}{|l|}{ Gender } \\
\hline Male & 21 & 49 & 2.368 & 0.124 \\
\hline Female & 18 & 74 & & \\
\hline \multicolumn{5}{|l|}{ Age, years } \\
\hline$>60$ & 5 & 15 & 0.011 & 0.918 \\
\hline$\leq 60$ & 34 & 108 & & \\
\hline \multicolumn{5}{|l|}{ ASA grade } \\
\hline$>2$ & 5 & 1 & 11.971 & 0.001 \\
\hline$\leq 2$ & 34 & 122 & & \\
\hline \multicolumn{5}{|l|}{ BMI } \\
\hline$>20$ & 31 & 108 & 1.682 & 0.195 \\
\hline$\leq 20$ & 8 & 15 & & \\
\hline \multicolumn{5}{|l|}{ BMI } \\
\hline$>25$ & 14 & 36 & 0.610 & 0.435 \\
\hline$\leq 25$ & 25 & 87 & & \\
\hline \multicolumn{5}{|l|}{ Tumor characteristic } \\
\hline Malignant & 13 & 28 & 1.750 & 0.186 \\
\hline Benign & 26 & 95 & & \\
\hline \multicolumn{5}{|l|}{ Location } \\
\hline Brainstem & 11 & 38 & 0.101 & 0.750 \\
\hline Adjacent to brainstem & 28 & 85 & & \\
\hline \multicolumn{5}{|l|}{ Tumor mass } \\
\hline$>100 \mathrm{~g}$ & 7 & 19 & 0.138 & 0.711 \\
\hline$\leq 100 \mathrm{~g}$ & 32 & 104 & & \\
\hline \multicolumn{5}{|l|}{ Partial resection } \\
\hline Yes & 12 & 16 & 6.534 & 0.011 \\
\hline No & 27 & 107 & & \\
\hline \multicolumn{5}{|l|}{ Surgical duration } \\
\hline$>5 \mathrm{~h}$ & 31 & 82 & 2.307 & 0.129 \\
\hline$\leq 5 \mathrm{~h}$ & 8 & 41 & & \\
\hline \multicolumn{5}{|l|}{ Smoking history } \\
\hline Yes & 12 & 13 & 9.258 & 0.002 \\
\hline No & 27 & 110 & & \\
\hline \multicolumn{5}{|l|}{ Basic lung disease } \\
\hline Yes & 4 & 3 & 4.377 & 0.036 \\
\hline No & 35 & 120 & & \\
\hline
\end{tabular}

$\mathrm{P}<0.05$ was considered to indicate a significant difference. BMI, body mass index; ASA, American Society of Anesthesiologists.

Table III. Logistic regression analysis on the independent risk factors influencing postoperative pulmonary complications.

\begin{tabular}{lcccc}
\hline Risk factor & B-value & $\begin{array}{c}\text { Substandard } \\
\text { error }\end{array}$ & OR value & $95 \%$ CI \\
\hline Partial resection & 1.108 & 0.468 & 3.028 & $(1.210-7.574)$ \\
Smoking history & 1.020 & 0.511 & 2.774 & $(1.019-7.555)$ \\
\hline
\end{tabular}

$\mathrm{P}<0.05$ was considered to indicate a significant difference. $\mathrm{B}$, binary logistic regression; OR, odds ratio; $\mathrm{CI}$, confidence interval. 
resection were independent risk factors of PPCs (Tables II and III).

\section{Discussion}

The present study has identified that, following elective craniotomy for patients with a brainstem or adjacent brainstem tumor, the incidence of PPCs was $23.9 \%$. The occurrence of PPCs was significantly associated with smoking history, instances of previous pulmonary disease, ASA class $>$ II and partial tumor resection. Smoking history and partial tumor resection were identified as independent risk factors of PPCs. To the best of our knowledge, this is the first report identifying partial resection as an independent risk factor.

The good, general condition of the patients that were enrolled in the present study indicates the uniformity of the population. There were 162 patients with normal preoperative hepatic and renal function, normal serum albumin and blood sugar levels, no serious cardiopulmonary insufficiencies (only six cases of patients with ASA class III, four cases of COPD and two cases of heart disease) and no histories of malignant tumors. All of the tumors were located in the narrow position of the brainstem or adjacent to the brainstem. All surgical procedures were performed under intravenous anesthesia, without the use of long-acting muscle relaxants and were performed at the same incision position by a team of three surgeons.

Due to the abovementioned reasons, the present study may avoid the confounding factors regarding PPCs and may, therefore, enable improved understanding of the associated risk factors of brain tumor patients with regard to PPCs. The present study has shown that partial tumor resection is an independent risk factor of PPCs, with subtotal and near subtotal resection surgery being associated with the highest incidence of PPCs. Thus, partial brainstem tumor resection has been identified as an independent risk factor of PPCs, which to the best of our knowledge, has not yet been reported in previous studies. This association may be due to residual tumor tissue, which continues to affect cerebral function. In addition, the present study has identified general risk factors of PPCs, including smoking history, which is a previously known independent risk factor of PPCs following lung, cardiac and abdominal surgery (9-15) and craniocerebral-associated surgical procedures (3). The present study demonstrates that smoking history is an independent risk factor of PPCs following brainstem and adjacent brainstem tumor surgery.

In contrast to previous studies $(1,9,10,16-18)$, the present study has shown that PPCs are not associated with age or surgery duration, however, this may be due to differences in sample size or other factors. Clinical history of previous craniotomy procedures for brainstem and adjacent brainstem tumors is usually considered a contraindication for subsequent elective craniotomies. Although the procedure can be performed, the risk is high, therefore, strict screening of patients is required. Screening should include monitoring of the general characteristics of the patients; however, despite postoperative monitoring, the risk of developing PPCs remains high $(24.1 \%)$. This is consistent with previous studies, which demonstrated that the likelihood of developing PPC following elective craniocerebral surgery is $11.2-24.6 \%(2,3)$.
Age is an independent risk factor of PPCs, which may be due to the age-associated decline of organ function. The data of the present study has shown that, despite variations in age, the function of each organ in the patients that were analyzed was normal, therefore, no significant correlation was identified between PPCs and age. Surgical procedures with a long duration have been considered to be an independent risk factor of PPCs. Qaseem et al (1) reported that the risk of pulmonary complications increases when surgery duration is $>4 \mathrm{~h}$. Sogame et al (3) reported that the probability of developing PPCs was significantly higher when surgery duration is $\geq 300 \mathrm{~min}$ when compared with procedures lasting $<300 \mathrm{~min}$. The present study showed that the surgery duration had no correlation with the development of PPCs following brainstem and adjacent brainstem tumor surgery. This may be due to the complexity of the surgery and a generally longer surgery duration. The mean surgery duration of patients in the present study was $6.06 \pm 0.16 \mathrm{~h}$, whereas the previous studies have reported mean surgery times of 3,4 or $5 \mathrm{~h}(6,17)$.

In conclusion, an ASA class $>$ II and chronic lung disease have previously been identified as risk factors and independent risk factors of PPCs of chest, abdomen and other types of surgery, as well as risk factors and independent risk factors of PPCs of craniocerebral procedures $(10,11,19-23)$. By contrast, in the present study, an ASA class $>$ II and chronic lung disease were identified to be risk factors, although not independent risk factors, of developing PPCs following surgical excision of brainstem or adjacent brainstem tumors. The reason for this difference is unknown, however, may be associated with the small sample size, as an ASA grade >II was observed in only six cases (all were ASA grade III), and chronic pulmonary disease was observed in only seven patients. Thus, future studies are required to clarify these discrepancies by expanding the sample size.

\section{References}

1. Qaseem A, Snow V, Fitterman N, et al; Clinical Efficacy Assessment Subcommittee of the American College of Physicians: Risk assessment for and strategies to reduce perioperative pulmonary complications for patients undergoing noncardiothoracic surgery: a guideline from the American College of Physicians. Ann Inten Med 144: 575-580, 2006.

2. Manninen PH, Raman SK, Boyle $\mathrm{K}$ and el-Beheiry $\mathrm{H}$ : Early postoperative complications following neurosurgical procedures. Can J Anaesth 46: 7-14, 1999.

3. Sogame LC, Vidotto MC, Jardim JR and Faresin SM: Incidence and risk factors for postoperative pulmonary complications in elective intracranial surgery. J Neurosurg 109: 222-227, 2008.

4. Landolfi JC, Thaler HT and Deangelis LM: Adult brainstem gliomas. Neurology 51: 1136-1139, 1998.

5. Murphy TF and Sethi S: Bacterial infection in chronic obstructive pulmonary disease. Am Rev Respir Dis 146: 1067-1083, 1992.

6. Pereira ED, Fernandes AL, da Silva Anção M, et al: Prospective assessment of the risk of postoperative pulmonary complications in patients submitted to upper abdominal surgery. Sao Paulo Med J 117: 151-160, 1999.

7. Prys-Roberts C, Nunn JF, Dobson RH, et al: Radiologically undetectable pulmonary collapse in the supine position. Lancet 2: 399-401, 1967.

8. Woodhead M, Blasi F, Ewig S, et al; Joint Taskforce of the European Respiratory Society and European Society for Clinical Microbiology and Infectious Diseases: Guidelines for the management of adult lower respiratory tract infections - full version. Clin Microbiol Infect 6: E1-E59, 2011. 
9. Kearney DJ, Lee TH, Reilly JJ, DeCamp MM and Sugarbaker DJ: Assessment of operative risk in patients undergoing lung resection. Importance of predicted pulmonary function. Chest 105: 753-759, 1994.

10. Brooks-Brunn JA: Predictors of postoperative pulmonary complications following abdominal surgery. Chest 111: 564-571, 1997.

11. Schuller D and Morrow LE: Pulmonary complications after coronary revascularization. Curr Opin Cardiol 15: 309-315, 2000.

12. Stéphan F, Boucheseiche S, Hollande J, et al: Pulmonary complications following lung resection: a comprehensive analysis of incidence and possible risk factors. Chest 118: 1263-1270, 2000.

13. Nakagawa M, Tanaka H, Tsukuma $\mathrm{H}$ and Kishi Y: Relationship between the duration of the preoperative smoke-free period and the incidence of postoperative pulmonary complications after pulmonary surgery. Chest 120: 705-710, 2001.

14. Møller AM, Villebro N, Pedersen T and Tønnesen H: Effect of preoperative smoking intervention on postoperative complications: a randomized clinical trial. Lancet 359: 114-117, 2002.

15. Canet $\mathrm{J}$ and Mazo V: Postoperative pulmonary complications. Minerva Anestesiol 76: 138-143, 2010.

16. Doyle RL: Assessing and modifying the risk of postoperative pulmonary complications. Chest 115:77S-81S, 1999.
17. Fuso L, Cisternino L, Di Napoli A, et al: Role of spirometric and arterial gas data in predicting pulmonary complications after abdominal surgery. Respir Med 94: 1171-1176, 2000.

18. Joo YH, Sun DI, Cho JH, Cho KJ and Kim MS: Factors that predict postoperative pulmonary complications after supracricoid partial laryngectomy. Arch Otolaryngol Head Neck Surg 135: 1154-1157, 2009.

19. Fish BW, Majumdar SR and McAlister FA: Predicting pulmonary complications after nonthoracic surgery: a systematic review of blinded studies. Am J Med 112: 219-225, 2002.

20. Beauregard CL and Friedman WA: Routine use of postoperative ICU care for elective craniotomy: a cost-benefit analysis. Surg Neurol 60: 483-489, 2003.

21. McAlister FA, Bertsch K, Man J, Bradley J and Jacka M: Incidence of and risk factors for pulmonary complications after nonthoracic surgery. Am J Respir Crit Care Med 171: 514-517, 2005

22. Jensen L and Yang L: Risk factors for postoperative pulmonary complications in coronary artery bypass graft surgery patients. Eur J Cardiovasc Nurs 6: 241-246, 2007.

23. Agostini P, Cieslik H, Rathinam S, et al: Postoperative pulmonary complications following thoracic surgery: are there any modifiable risk factors? Thorax 65: 815-818, 2010. 